\title{
28 Research Square \\ Text Feature Extraction of Myopia-related \\ Information on Social Media -A Study Based on the Text Sentiment and Statistical Evidence
}

\section{Zuyue Zhang}

College of Medical Informatics, Chongqing Medical University, Chongqing 400016

Mengyao Jiang

College of Medical Informatics, Chongqing Medical University, Chongqing 400016

Qingmao Rao

West China Second University Hospital, Sichuan University, Sichuan 610066

Haolin Wang

College of Medical Informatics, Chongqing Medical University, Chongqing 400016

Chunlv Ye

Nanping Sihai Elementary School, Nanping District, Chongqing 400016

Ting Cheng

Children's Hospital of Chongqing Medical University, Chongqing 400016

Xiaorong Hou ( $\nabla$ xiaoronghou@cqmu.edu.cn )

College of Medical Informatics, Chongqing Medical University, Chongqing 400016

\section{Research Article}

Keywords: Health information, Text feature, Text sentiment, Statistical evidence

Posted Date: February 16th, 2022

DOl: https://doi.org/10.21203/rs.3.rs-1255885/v1

License: (c) (i) This work is licensed under a Creative Commons Attribution 4.0 International License.

Read Full License 


\section{Abstract}

Background: In recent years, health information has become easily available on in social media. However, most of the available information lacks a persuasive effect. Texts providing information in health science on social media may affect how readers are convinced of the evidence presented. The purpose of this study is to examine health information on myopia prevention in WeChat using text sentiment indicators and to assess the extent of use of statistical evidence.

Methods: A Web crawler is employed to collect health science information data from the WeChat official account. A total of 4,645 myopia prevention texts of children in WeChat were collected, and 813 valid texts were included in the study. A paired-samples T-test was used in the study. The text sentiment and use of statistical evidence of health science information were extracted by analysing the text features.

Results: Results show higher use of positive words as compared to negative words $(P<0.01)$. Statistical evidence was used in over $96 \%$ of the text. Suggestions for the development of health science information in terms of text sentiment and statistical evidence are also proposed.

Conclusions: This study investigated the emotional features of text and use features of statistical evidence of health science information in WeChat. The study found that producers of information related to myopia prevention prefer positive emotions and use statistical evidence in most information.

\section{Introduction}

The rapid development of network technology has resulted in the Internet becoming a significant source of health information for children and adolescents. They rely on schools, hospitals and government departments for health information. However, the quality of health information varies, and repetitive information and a lack of information review process exist[1]. With the emergence of extensive online health information, poor quality information can cause numerous problems that, may even have negative effects[2]. Therefore, online health education should be improved[3].

The text features of information affect the dissemination of information. Text features analysis refers to the representation of text and the selection of its feature items. Different information features affect the transmission effect[4]. Wen Yong took Twitter as the research object and classified short media texts by extracting 16 external features based on tweet format [5]; Zhang Pengwei posited that the different distributions of text features under various themes of microblogs have distinct influences on communication effects[4]. Gondy Leroy determined that the current health science information on the Internet is overly complex and difficult to understand. Another study found that grammar, words and health topics affect the level of difficulty in understanding health information[6]. However, studies that focus on Chinese text features of new media involving health are scarce and studies that combine text features with the persuasive effect of health science information have also been very limited. 
Ultimately, the current study aims to contribute to the guidance on the use of emotional text and statistics in health science information representing social media by children and adolescents. This study can serve as a reference in the formulation of popular myopia prevention health education information on the Internet to improve the persuasive effect of myopia prevention information on children and adolescents, improve their awareness of healthy eye use, and reduce the prevalence of myopia (the prevalence of myopia among children and adolescents in China was 53.6\% in 2018).

\section{Evaluating Indicator}

Our previous research have has shown the higher acceptance of gain-framed messages among participants as compared to loss-framed messages hat under the theme of the issue of mental health[7] . Another study focused on , and Under the theme of food safety, and found that loss-framed messages were more acceptable than gain-framed messages[8]. . We also found that positive emotions and shortterm behavioural outcomes of stress can improve the acceptance of information by children's caregivers within the context of childhood obesity[9]. In a recent study, we investigated the influence of text sentiment and evidence type on the persuasive effect of myopia prevention messages, which is the basis of this study[10]. We conducted a questionnaire survey and the results revealed that changing the text sentiment and different types of evidence will affect the persuasive effect of information. In the study, we conducted a questionnaire survey on 1493 primary school students aged 9-13. Through the analysis of the subjects' feedback on information materials, we found the persuasion effect of statistical evidence is lower than that of non-statistical evidence, and the difference is statistically significant. Information has the best persuasion effect when non-statistical evidence is used and the positive results of doing something are emphasized. Therefore, in the current research, 'text sentiment' and 'statistical evidence' are used as evaluating indicators to explore the text features of health science information, and lay the foundation in determining the methods for improving the persuasion effect of health science information through text features.

\section{Text sentiment}

Text sentiment analysis also referred to as opinion mining, is the method of analyzing, processing, induction and reasoning[11]. It can be divided into word-, sentence- and text levels based on the different analysis units [12]. The original sentiment analysis refers to the analysis of words with emotions[13], such as 'good', 'delicious' and 'happy' to represent the positive sense, and 'bad' , 'ugly' and 'corruption' to represent the negative sense. The sentiment of the text will affect the transmission of information. Amos Tversky and Daniel Kahneman found that preference changes when the same question is expressed in different ways[14]. Rothman et al. believed that compared to positive expression, the negative expression had a better effect in promoting detection behaviour, but the result was the opposite in promoting preventive behaviour[15]. The finding of Lannotti and RIET confirmed this view[16]. However, some scholars have offered different conclusions, such as Gerend and Lithopoulos, who found that negative expression is more convincing in vaccination (prevention) behaviour and the willingness to participate in 
physical activities of multiple sclerosis patients[17, 18]. Hogenboom and Alexander determined that sentiment analysis can be guided, and that a deep analysis of the rhetorical structure of a text can yield better understanding of the conveyed sentiment in relation to an entity of interest. Therefore, in this study, we take word-level sentiment as one of the evaluating indicators of text features.

\section{Evidence Types}

Different evidence types in information will affect the persuasive effect of information, including exemplar and statistical evidence[19]. Typically, statistical evidence specifies quantity evidence, which refers to information expressed in mathematical terms[20]. Previous studies have found that exemplar evidence often affected attitudes more effectively than statistical evidence[21-23]. The effect of narrative evidence is greater than that of statistical evidence in the patient education on anticoagulant medication and in increasing men's perceived risk of infection with HBV and intention to obtain vaccination[24, 25]. Another study determined that statistical evidence is more persuasive than anecdotal evidence within the context of an argument by generalization[26]. Jiang found that participants had a higher degree of pleasure and arousal to statistical titles as compared with exemplar titles [27]. Ran used the persuasive effect theory in exploring the dissemination of cervical cancer health information and concluded that low threat statistical information had a more significant effect on an individual's willingness to prevent than high threat exemplar information with a high fear effect[28]. Limon revealed that narrative evidence and statistical evidence are more convincing than without any evidence, they are all effective ways to improve the persuasiveness of information [19]. Changing the evidence type of information can affect the persuasion effect of information. Thus, this study intends to include statistical evidence as to the second evaluating indicator.

\section{Research Topics}

'Healthy China Action (2019-2030)' has reported that the overall myopia rate of children and adolescents in 2018 was 53.6\%. In 2018, eight departments, including the Ministry of Education and the National Health Commission jointly issued an implementation plan for the comprehensive prevention and control of myopia in children and adolescents, thereby raising the prevention and control of myopia in children and adolescents to a strategic goal. Many hospitals, schools and disease control departments in China use WeChat to issue health information. Therefore, we took the prevention of myopia in children and adolescents as the research topic and explored the current situation of text features of health science information. In this study, we conducted a cross-sectional study on the text features of myopia prevention information from February 2021 to May 2021. Textual information on myopia prevention was collected from WeChat, and the two text features of text sentiment and statistical evidence were analysed in the health science information. The design process of this study is shown in Figure 1.

\section{Data collection}


A web crawler was used to collect data from November 2020 to March 2021. A total of 4,645 myopia prevention messages published after 2015 were collected. The inclusion and exclusion criteria for this step are as follows:

1. Information unrelated to myopia prevention was excluded. For example, notification information released by the information publisher, such as notification of the time and place of myopia prevention-related activities (there is no health education information related to myopia prevention).

2. Information for marketing purposes, such as advertisements issued by the glasses dealer, was excluded.

3. Highly repetitive information was excluded. For example, new documents released by government departments will be reprinted by many WeChat accounts (resulting in duplicate information, which were excluded).

4. Information that lacked a scientific basis was eliminated. For example, disinformation on myopia prevention. A pediatrician was involved in information screening. We excluded information that lacked a scientific basis or information that does not affect the prevention of myopia

After screening and cleaning, 813 valid data were obtained, and the data qualification rate was $17.5 \%$.

\section{Research Method}

In this study, natural language processing technology was used to handle data, and Jieba was used for word segmentation. The data were processed using MS Excel before being entered in into SPSS25.0 software (IBM Corporation, Armonk, NY, US) for data analysis.

The text of the WeChat official account platform is mostly long, whereas the traditional text sentiment analysis often focuses on short texts, such as micro-blog text. The long text contains more sentences and numerous emotional words. Therefore, of the method for short text sentiment analysis may not be suitable for this study. Therefore, this study, 1) considered the method of emotional words (based on the Chinese emotional vocabulary ontology of Dalian University of Technology). All the positive and negative words from the text were extracted. This study investigated the preferences of information makers in their choice of text sentiment when assigning information by analyzing the use frequency of positive and negative words in a long text.

2) Many numbers are used in a long text, including serial and telephone numbers, which are irrelevant to this study. Hence, to eliminate the influence of meaningless numbers on the research results, we consider the number of uses of quantity unit words as the number of uses of statistical evidence because the uses of quantity unit words and numbers appear simultaneously and form meaningful data evidence.

In terms of the frequency and percentage of classified variables, the mean and standard deviation of continuous variables were calculated. Paired sample t-test was used to analyze the differences in the use of positive and negative emotional words. A P-value less than 0.05 was statistically significant. 


\section{Research Results}

\section{Results of the text sentiment analysis}

We analyzed the text sentiment of myopia prevention information and referred to the 'positive and negative words' database in the Chinese emotion vocabulary ontology database of the Dalian University of Technology. Then, as shown in Table 1, emotional words in each text were extracted and analysed. We then counted the words that appeared in the text that can be found in the emotion dictionary as shown in Figure 2. We analyzed and compared the 'number of positive words used for every thousand words' and 'the number of negative words per thousand words' to avoid errors caused by the different numbers of texts. The frequency comparison for positive and negative words for every thousand words is shown in Figure 3. Tables 2 and 3 indicate that in terms of text sentiment in the information related to myopia prevention, the average number of commendatory words per thousand words was 13.9 , while that of negative words was 5.5. The results indicate that the frequency of use of positive words was considerably higher than that of negative words, and the difference was significant $(P<0.01)$ indicate by the paired sample t-test analysis. The minimum number of commendatory words per thousand words is 2.85 and the maximum is 35.45 , while the minimum number of derogatory words per thousand words is 0 and the maximum is 18.18 .

Table 1. Examples of positive and negative words

\begin{tabular}{ll} 
Positive & Negative \\
\hline happy & bad \\
\hline healthy & damage \\
\hline beautiful & Insufficient \\
\hline effective & serious \\
\hline security & blind \\
\hline cosy & lack \\
\hline beneficial & reproach \\
\hline progress & Crying \\
\hline care & adverse \\
\hline guarantee & fake \\
\hline rich & virus
\end{tabular}

Table 2. Use of emotional words for every thousand words $(n=813)$ 


\begin{tabular}{|c|c|c|c|c|}
\hline & Minimum & Maximum & Mean & Std.Deviation \\
\hline $\begin{array}{l}\text { Commendatory words } \\
\text { for every thousand words }\end{array}$ & 2.851 & 35.452 & 13.922 & 5.654 \\
\hline $\begin{array}{l}\text { Derogatory words } \\
\text { for every thousand words }\end{array}$ & 0 & 18.182 & 5.549 & 3.007 \\
\hline
\end{tabular}

Table 3. Paired sample $t$-test $(n=813)$

\begin{tabular}{|c|c|c|c|c|c|c|c|c|}
\hline & \multirow[t]{2}{*}{ Mean } & \multirow[t]{2}{*}{ Std.Deviation } & \multirow{2}{*}{$\begin{array}{l}\text { Std.Error } \\
\text { Mean }\end{array}$} & \multicolumn{2}{|l|}{$95 \% \mathrm{Cl}$} & \multirow[t]{2}{*}{$t$} & \multirow[t]{2}{*}{ df } & \multirow[t]{2}{*}{ Sig. } \\
\hline & & & & Lower & Upper & & & \\
\hline $\begin{array}{l}\text { Positive } \\
\text { words (13.922) - } \\
\text { Negative } \\
\text { words (5.549) }\end{array}$ & 8.373 & 6.759 & 0.237 & 7.908 & 8.838 & 35.321 & 812 & 0.000 \\
\hline
\end{tabular}

\section{Results of statistical evidence analysis}

To identify the use of statistical evidence in myopia prevention texts, we counted the use of quantitative unit words and avoid the influence of meaningless numbers on the results. Considering that the difference in children's understanding of Chinese and English unit words, we split the use of unit words into Chinese and English unit words and employed, Python to extract and count the use of words of quantitative units. The process of single sentence unit extraction is shown in Figure 4. Tables 4 and5 show that only $3 \%$ of the texts (24) use English quantitative unit words. Meanwhile, $96.4 \%$ of the texts (784) use Chinese unit quantifiers, with an average of 7.55 per text, and the quartile is $(4,10)$. The number of Chinese unit quantifiers is concentrated between 4 and 10. Figure5 shows the maximum number of Chinese unit quantifiers is 45 , and the minimum is 0 . Based on the study design, over $96 \%$ of myopia prevention-related texts use data evidence, and the maximum value is 45 .

Table 4. Frequency of unit quantifiers in Chinese and English $(n=813)$

\begin{tabular}{|lll|}
\hline & frequency & Percentiles \\
\hline Chinese units & 784 & 96.4 \\
\hline English units & 24 & 3 \\
\hline
\end{tabular}

Table 5. Use of unit words of quantity in Chinese and English $(n=813)$ 


\section{Number of Chinese units Number of English units}

\begin{tabular}{llll}
\hline Mean & & 7.55 & 0.06 \\
\hline Median & 7 & 0 \\
\hline Percentiles & 25 & 4 & 0 \\
\hline & 50 & 7 & 0 \\
\hline & 75 & 10 & 0
\end{tabular}

Table 6 shows that the top 10 quantity unit words are 'hour' (2,271 times), 'minute' (2,192 times), 'centimetre' (613 times), 'metre' (461 times), 'year' (438 times), 'month' (378 times), 'second' (194 times), 'foot' (157 times), 'cm' (32 times) and 'Chi' (31 times). The words 'hour and minute' account for $65.6 \%$ of the top 10 , while the total number of length units is only $19.1 \%$.

Table 6. Statistics of quantity unit word frequency

\begin{tabular}{|c|c|c|}
\hline Sort results & Unit & Word frequency (Percentiles) \\
\hline 1 & $\mathbb{Q}$ (Hour) & $2271(33.56 \%)$ \\
\hline 2 & $\mathbb{Q} \mathbb{Q}($ Minute) & $2192(32.39 \%)$ \\
\hline 3 & 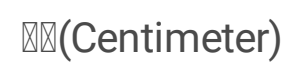 & $613(9.06 \%)$ \\
\hline 4 & $\mathbb{\nabla}$ (Meter) & $461(6.81 \%)$ \\
\hline 5 & $\bigotimes($ Year) & $438(6.47 \%)$ \\
\hline 6 & $\rrbracket$ (Month) & $378(5.59 \%)$ \\
\hline 7 & $\mathbb{\nabla}($ Second $)$ & $194(2.87 \%)$ \\
\hline 8 & $\mathbb{Q} \mathbb{Q}($ Foot $)$ & $157(2.32 \%)$ \\
\hline 9 & $\mathrm{~cm}$ & $32(0.47 \%)$ \\
\hline 10 & $\rrbracket($ Chi,3.3cm) & $31(0.46 \%)$ \\
\hline Total & & $6767(100 \%)$ \\
\hline
\end{tabular}

\section{Discussion And Suggestions}

\section{Sentiment of the text}

The results of the analysis on the sentiment of myopia prevention-related texts indicate a significantly higher use of positive emotion than negative emotion (13.9 vs $5.5, P<0.01)$, which are consistent with our previous research. In the previous research, the persuasion effect had the best effect when positive sentiment and non-statistical evidence were used simultaneously. Thus, the current findings in terms of 
the emotional tendency of myopia prevention-related texts are in line with our expected emotional features. the use of positive words is also much higher than the use of negative words, thereby indicating that health information makers prefer to use positive emotions when formulating health information. Some scholars found that children are more receptive to positive and encouraging methods, which coincide with the results of this study and that of the previous study by our team [29].

Thus, the use of more positive emotional words could contribute to improving the persuasive effect of information when developing health information.

Use of statistical evidence

The analysis of the statistical evidence indicated that most of the texts $(96.4 \%)$ used Chinese quantitative unit words, with a number between 4 and 10 , and a maximum value is 45 . This result differs from previous research in that in the previous study, the persuasion effect was the highest when positive sentiment and non-statistical evidence are used simultaneously. The current findings may be related to the theme and topic of the study. Among the relevant pieces of information on myopia prevention, myopia prevention had a strong correlation with screen use time, outdoor activity time and reading distance[30]. These factors are closely related to the use of numbers, and therefore, in this kind of text, the use of statistical evidence cannot be avoided. In terms of the use of units, we also found a special expression ("the length of a fist"), which differs from the traditional expression. We think that this analogical expression is easier for children to understand and remember because this type of unit word uses an object to represent length.

An analysis of the statistics of the frequency of quantity unit words shows that the most frequently used unit words are 'hour' and 'minute', which account for $65.6 \%$ of the total number of the top 10 unit words used and correspond to eye use time and outdoor activity time. And total number of length unit words in the top 10 unit words only accounts for $19.1 \%$ of the total number. It corresponds to the description of eye distance. The results indicate that in the design of myopia prevention-related texts, health information makers pay the most attention to the amount of time of eye use followed by the distance of eye use. When statistical evidence must be used in some health topics, an analogy can be used to improve the acceptance effect of information. For instance, in some information, "the length of a fist" was used to describe the exact distance and the time of an animation episode was used to represent about 30 minutes. Reducing the use of numbers and replacing it with an analogy can make the information easier to understand and contribute to the better expression of the of information.

\section{Limitations}

The myopia-related information selected in this study represent all the health information available online. Therefore, further studies that focus on the different types of health information should be conducted to form a better understanding and provide more suggestions for the development of health information. The current study investigated a specific method for exploring text sentiment and the use of 
statistical evidence of different types of health science information on social media. The myopia prevention information collected in this study also contains videos and pictures. However, because of certain limitations of these unstructured data analyses and the main information carrier being text, hence, the current study only included text data. Although other factors, such as internet penetration, relevant risk perceptions, individual involvement education, eHealth literacy, etc. may also influence the transmission effect of health information, these moderating variables are included in the current study.

\section{Practical implication}

This study investigates the current situation of text features of online information on myopia prevention. The results of this study can be used as a reference in the formulation of online myopia prevention information and in exploring the various means for improving the efficiency of health information dissemination. Moreover, the observation indicators of this study can be used as a reference in future studies on the text features of information in other public health fields. The results can also be employed in more scenarios for empirical research and applied to more health themes and different populations.

\section{Conclusion}

This study explored the current situation of the text features of health information and found that the use of positive sentiment in WeChat was significantly higher than the use of negative emotion, and most of the texts used statistical evidence. In addition, in terms of the consciousness of the informant formulating information, the most important factor is the time of eye use followed by the distance of eye use. Compared with our previous studies the use of positive emotions helped improve the persuasive effect of information. Considerable statistical evidence in information on the prevention of myopia in children can be found and we can use analogical methods to express exact figures, such as employing the phrase, "the length of a fist" to indicate the distance (about $10 \mathrm{~cm})$. The persuasive effect of information can increase if more positive emotions are used in health science information and the use of statistical evidence is reduced or more specific quantity units are used.

\section{Declarations}

\section{i) Ethics approval and consent to participate}

The study protocol was approved by the Ethics Committee of Chongqing Medical University (The record number is 2018011). All methods are implemented in accordance with relevant guidelines and regulations.

\section{ii) Consent for publication}

Not applicable. 
All data generated or analysed during this study are included in this published article [and its supplementary information files].

\section{iv) Competing interests}

The authors declare that they have no competing interests.

\section{v) Funding}

This research was funded by the Ministry of Education in China Project of Humanities and Social Sciences (Research on the mechanism and intervention countermeasures of framing effects in the formation of adolescent health literacy, grant number 20YJCZH043); the Intelligent Health Knowledge Discovery and Information Service in Chongqing Medical University (grant number ZHYX2019006); Chongqing Medical University, China, the Intelligent Medical Foundation of Chongqing Medical University (grant number YJSZHYX202016); the College of Medical Informatics, Chongqing Medical University, China, Student Research and Innovation Experiment Project (grant number 2019C009); Chongqing Municipal Education Commission, China, Chongqing Graduate Scientific Research Innovation Project (grant number CYS20225)

\section{vi) Authors' contributions}

X.H., Z.Z., C.Y., T.C., and M.J. performed the experiments, Z.Z. and M.J. analyzed the data and wrote the paper. Q.R. assisted in the data analysis. X.H. and H.W. helped draft the manuscript. Z.Z. and M.J. contributed equally to this work. All authors have read and approved the final manuscript.

\section{vii) Acknowledgment}

Not applicable.

\section{References}

1. Li-rong S, Na Q, Qun Z: Thinking on the Information Quality Problems of Network Health Information Transmission Journal of Medical Intelligence 2014, 35(10):8-12.

2. Ju H: Study on the Online Health Information Communication Effect: In The Case Of Online Selfdiagnose Behavior. master. University of Electronic Science and technology; 2015.

3. Xing A, Dian X, Jiao M, Xiao M, Yang Y: Research on health information communication based on practical experience of Microblog Chinese Journal of Health Education 2016, 32(2):141-143.

4. Pengwei Z, Hongli L, Chengdong Z, Shuhang C, Lijuan Y: Content Body and Correlative Field Based Analysis on Differences of Micro-blog Text Features' Effects to Information Dissemination. Information Research 2016(10):5-11.

5. Yong W: A Study For Classifying Short Text In Social Media. master. University of Electronic Science and technology; 2018. 
6. Leroy G, Eryilmaz E, Laroya BT: Health information text characteristics. AMIA Annual Symposium proceedings / AMIA Symposium AMIA Symposium 2006, 2006:479.

7. Bai, Qingmao, Rao, Zhengjie, Cai, Yalan, Tingting, Zumin, Shi, health MJFip: Effects of Goal-Framed Messages on Mental Health Education Among Medical University Students: Moderating Role of Personal Involvement. 2019, 7:371-371.

8. Bai, Li, Cai, Zhengjie, Yalan, Tingting, Sharma, Manoj, Shi, Research ZJIJoE et al: Personal Involvement Moderates Message Framing Effects on Food Safety Education among Medical University Students in Chongqing, China. 2018.

9. Rao Q, Bai L, Yalan LV, Abdullah AS, Brooks I, Xie Y, Zhao Y, Hou X: Goal-Framing and TemporalFraming: Effects on the Acceptance of Childhood Simple Obesity Prevention Messages among Preschool Children's Caregivers in China. International Journal of Environmental Research Public Health 2020, 17(3).

10. Zuyue Zhang, Yalan Lv, Qingmao Rao, Chunlv Ye, Ting Cheng, Mengyao Jiang, Li Bai, Hou X: Framing Effects and Evidence Type: Influence on the Persuasive Effect of Myopia Prevention Messages among Elementary School Students in China. Frontiers in public health 2021.

11. Church RT, Wilbanks CJGS, Pub: Values and policies in controversy : an introduction to argumentation and debate. 1986.

12. Wei W, Xiangyang, Qian C: Survey on Chinese text sentiment analysis Journal of Computer Applications 2011, 31(12):3321-3323.

13. Vasileios Hatzivassiloglou, McKeown KR: Predicting the Semantic Orientation of Adjectives. Proceedings of the Acl 1997:174-181.

14. Tversky A, Kahneman D: The framing of decisions and the psychology of choice. Science (New York, NY) 1981, 211(4481):453-458.

15. Rothman AJ, Salovey P: Shaping perceptions to motivate healthy behavior: the role of message framing. Psychological Bulletin 1997, 121(1):3-19.

16. Lannotti RJ, Finney LJ, Sander AA, De Leon JM: Effect of clinical breast examination training on practitioner's perceived competence. Cancer detection and prevention 2002, 26(2):146-148.

17. Lithopoulos A, Bassett-Gunter RL, Ginis KAM, Latimer-Cheung AE: The Effects of Gain- versus LossFramed Messages Following Health Risk Information on Physical Activity in Individuals With Multiple Sclerosis. Journal of health communication 2017, 22(6):523-531.

18. Gerend MA, Shepherd JE: Using message framing to promote acceptance of the human papillomavirus vaccine. Health psychology : official journal of the Division of Health Psychology, American Psychological Association 2007, 26(6):745-752.

19. Limon MS, Kazoleas DC: A comparison of exemplar and statistical evidence in reducing counterarguments and responses to a message. Communication Research Reports 2004, 21(3):291-298.

20. Church RT, Wilbanks C: Values and policies in controversy:an introduction to argumentation and debate: Values and policies in controversy:an introduction to argumentation and debate; 1986. 
21. Borgida E, Nisbett RE: The Differential Impact of Abstract vs. Concrete Information on Decisions1. Journal of Applied Social Psychology 1977, 7(3):258-271.

22. Brosius HB, Bathelt A: The Utility of Exemplars in Persuasive Communications. Communication Research Reports 1994, 21(1):48-78.

23. Hamill R, Wilson DC, Nisbett RE: Insensitivity to sample bias: Generalizing from atypical cases. Journal of Personality Social Psychology 1980, 39(4):578-589.

24. de Wit JB, Das E, Vet R: What works best: objective statistics or a personal testimonial? An assessment of the persuasive effects of different types of message evidence on risk perception. Health psychology : official journal of the Division of Health Psychology, American Psychological Association 2008, 27(1):110-115.

25. Mazor KM, Baril J, Dugan E, Spencer F, Burgwinkle P, Gurwitz JH: Patient education about anticoagulant medication: Is narrative evidence or statistical evidence more effective? Patient Education and Counseling 2007, 69(1):145-157.

26. Hoeken H, Hustinx L: When is Statistical Evidence Superior to Anecdotal Evidence in Supporting Probability Claims? the Role of Argument Type. Human Communication Research 2009, 35(4):491510.

27. Tingting J, Qian G, Yaping X, EnMei S: Evidence Type Influence on the Selection of Online Health Information Titles:Eye-Tracking Experiment and Implications Library and Information Service 2020, 64(19):61-70.

28. Hua R, Shupei G: The Influence of Information Characteristics and Organization Modeon the Reception of Audiencevia Health Communication Perspective: A Study of Cervical Cancer Prevention. Journalism and Communication Review 2018, 0(5).

29. yaju Y: The application of stimulating language in primary school Chinese Teaching. Science and Technology Innovation Herald 2015(7):147-148.

30. Sun LL, Qi LL, Ji TJIES: Correlation analysis of electronic products with myopia in preschool and school aged children. 2016, 16(2):382-385.

\section{Figures}




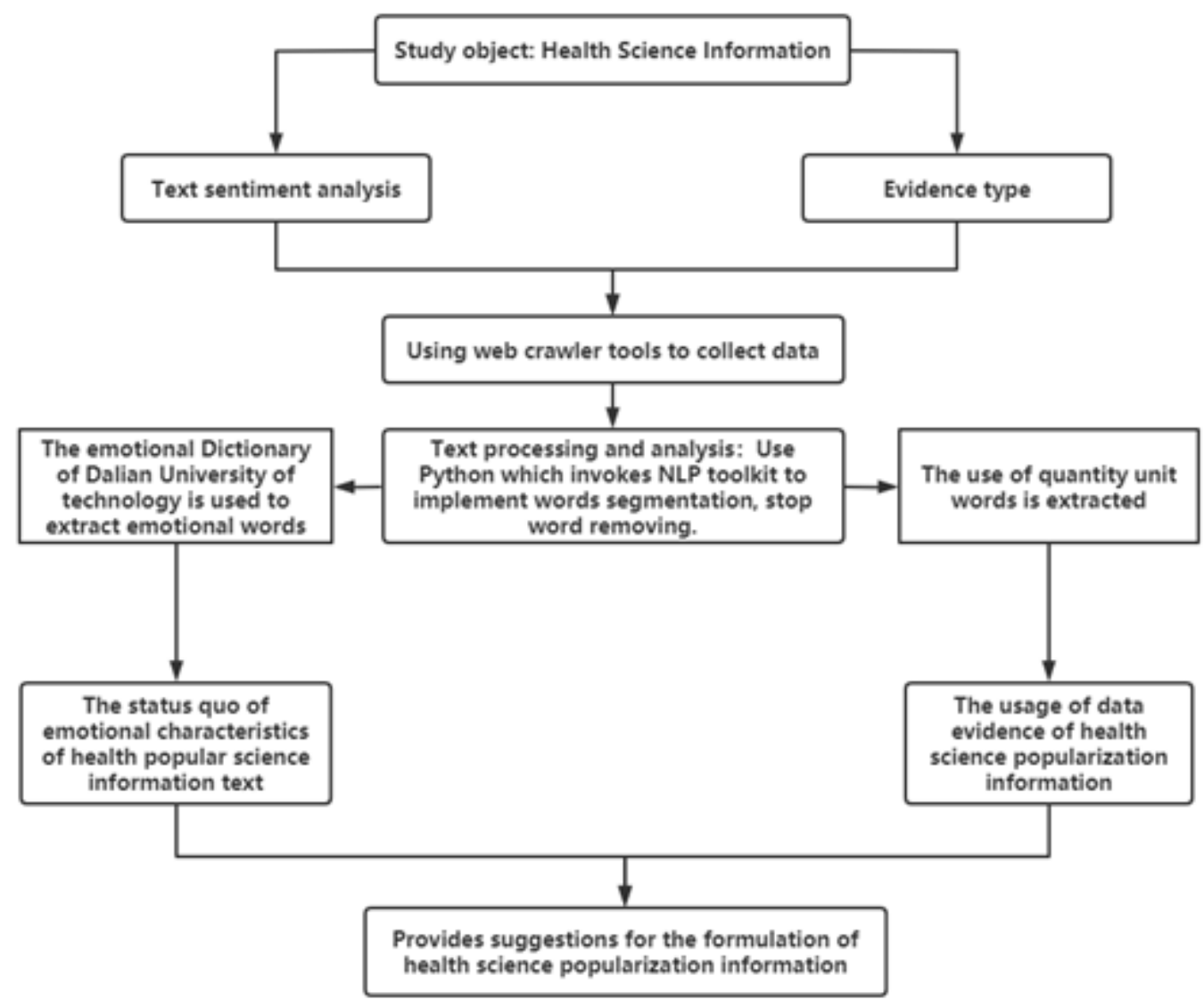

Figure 1

Research flow chart

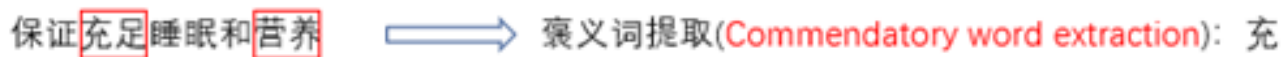
足(Enough)、营养(Nutrition)

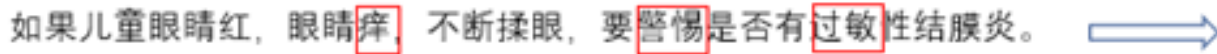
贬义词提取(Derogatory word extraction)：㾕(Itch)、警惕(Vigilant)、过敏(Allergy)

\section{Figure 2}

Process of emotion word extraction 


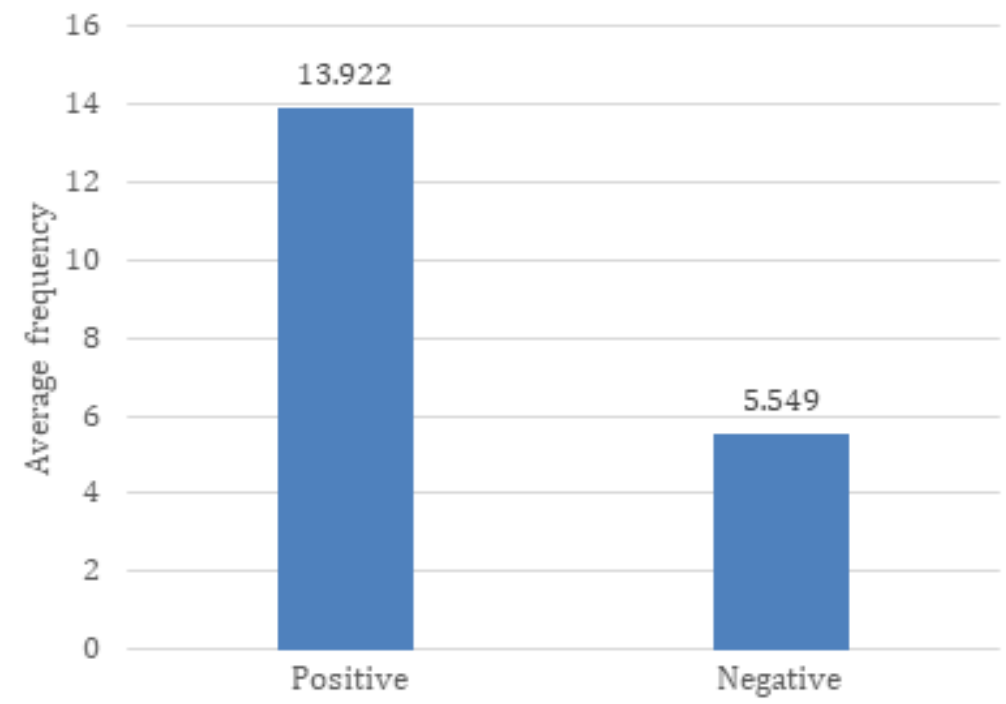

Figure 3

Frequency of positive and negative words per thousand words

保证一闭有 2 个小时充足的活动时间 $\Longrightarrow$ 单位提取(Unit extraction)：天 (Day)、小时(Hour)

家中的书桌椅应该调整好高度，读书写字的时候保持“三个一”，手离笔尖一団、离书 本一回，距书桌一搼。 $\Longrightarrow$ 单位提取(Unit extraction): 寸(Cun, About 3.3 centimeter)、尺(Chi, about 33.3 centimeter)、拳 (The length of a punch, about 10 centimeter)

Figure 4

Unit extraction process 


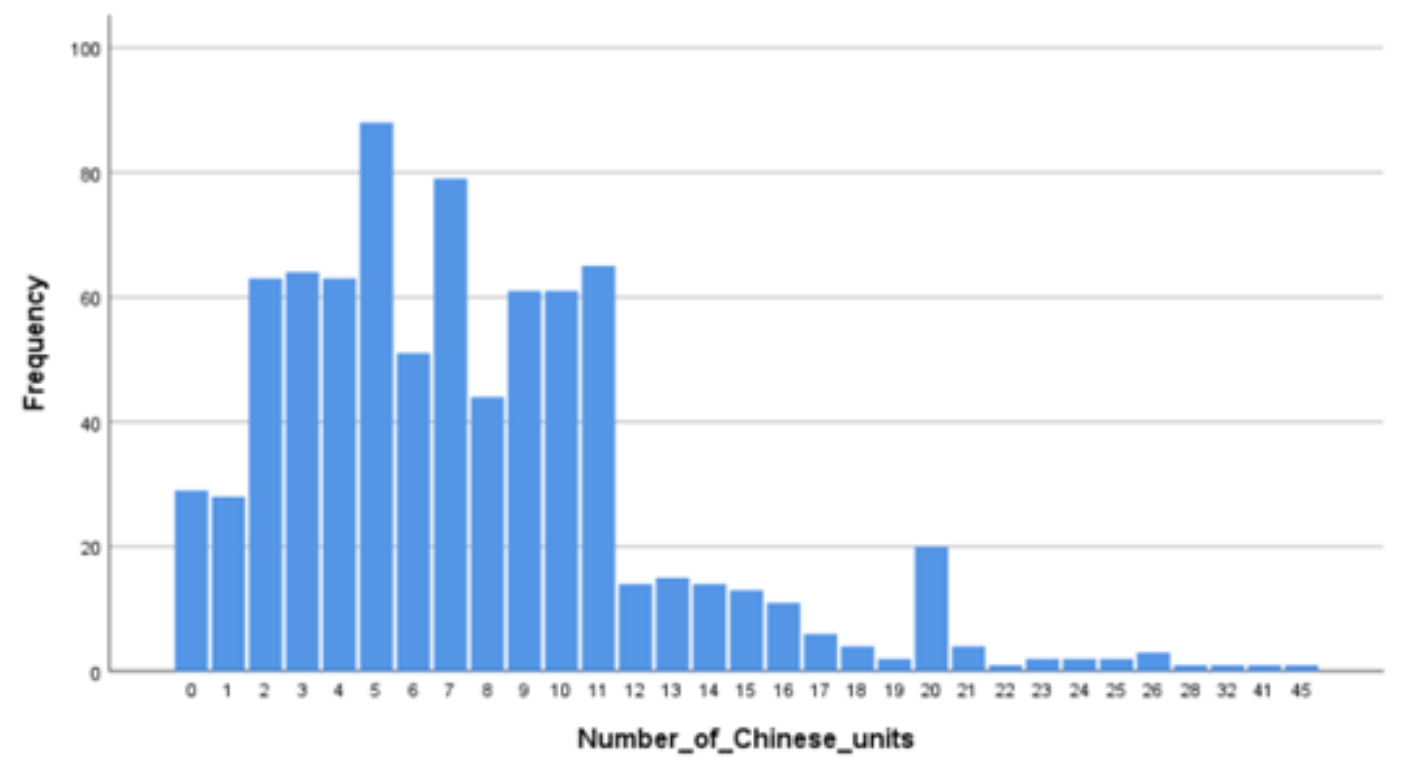

Figure 5

Use of unit quantity words in Chinese 